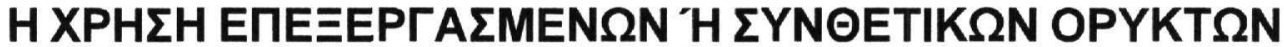 $\Sigma T H N$ ПAPA KKEYH TPOФIM $\Omega N$, $\$ A P M A K \Omega N$,

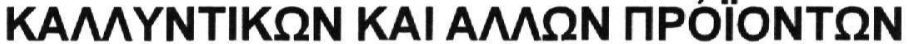

Tбıрантібіпs A.

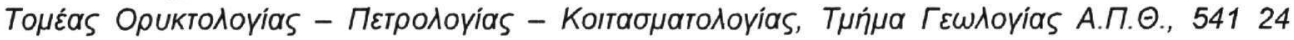

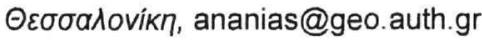

\section{ПЕРІ $\mathrm{H} \Psi \mathrm{H}$}

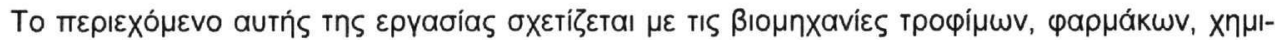

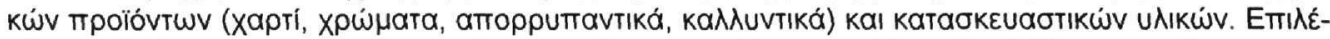

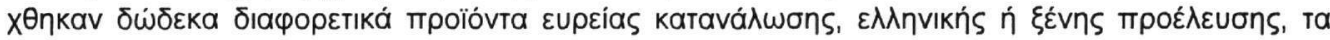

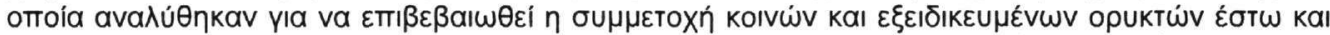

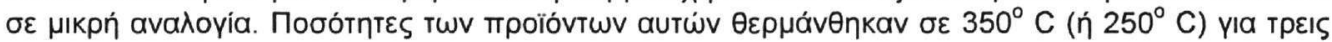

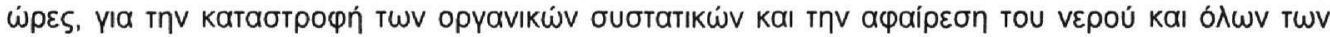

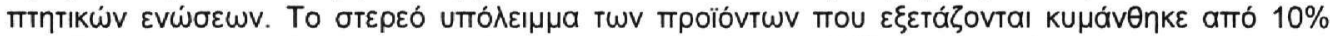

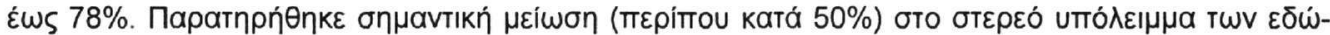

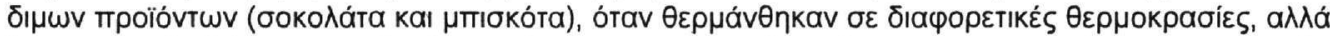

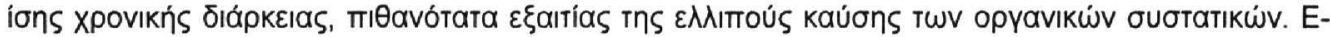

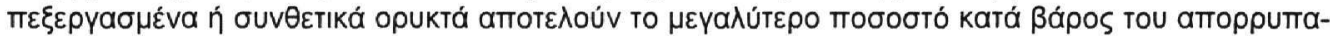

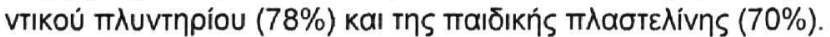

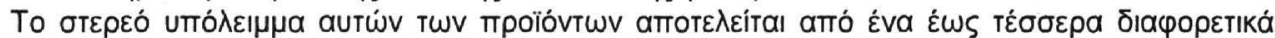

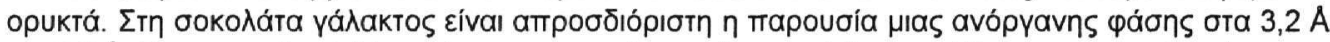

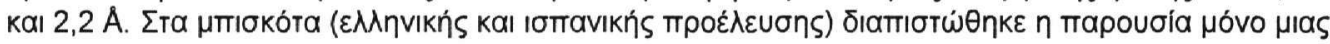

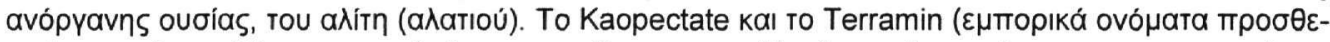

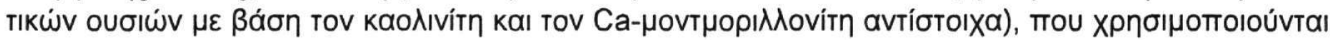

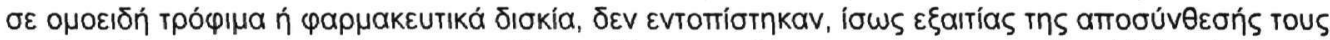

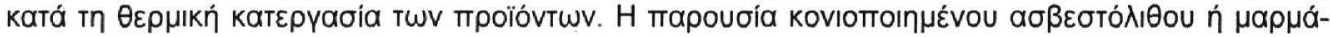

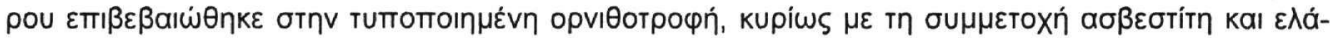

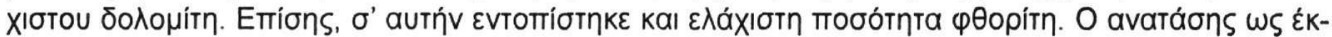

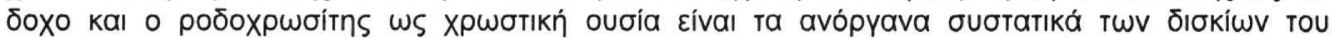

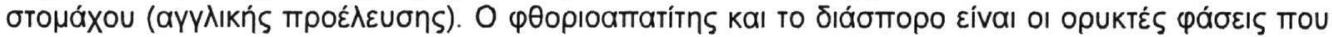

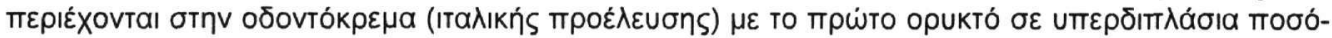

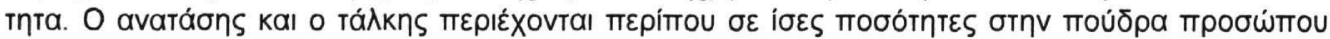

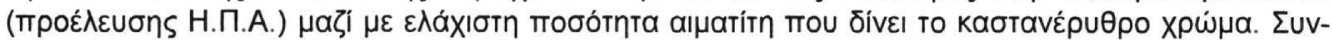

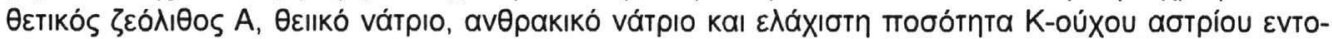

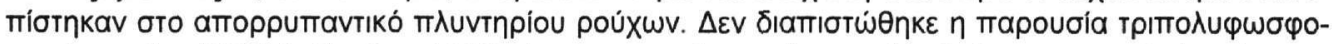

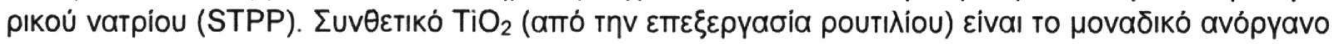

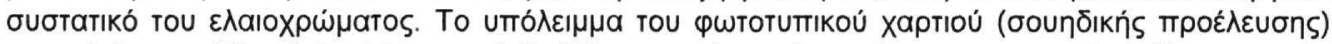

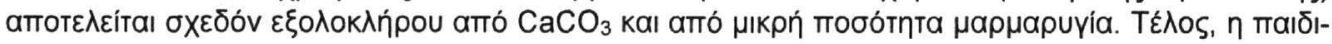

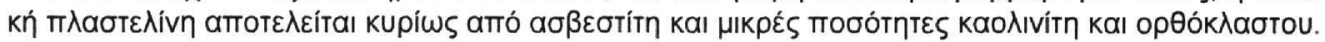

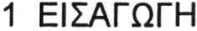

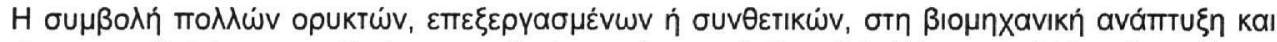

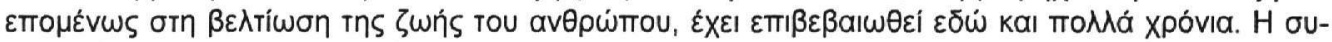

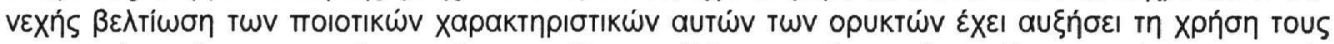

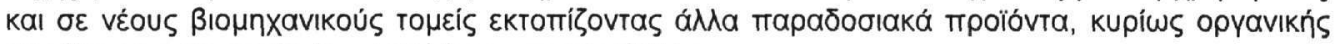

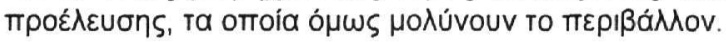




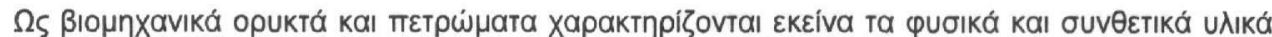

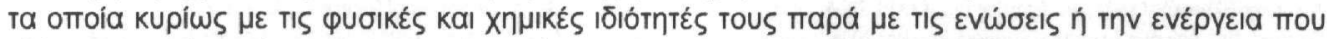

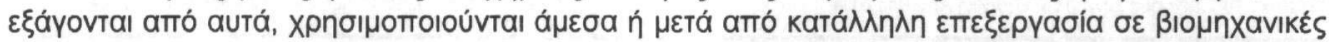

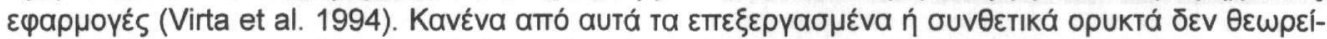

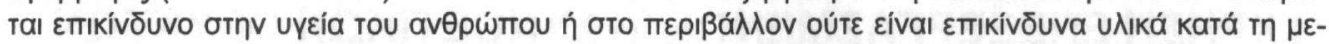

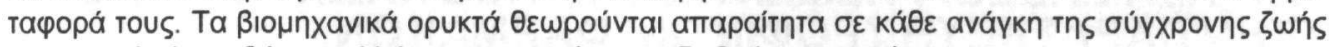

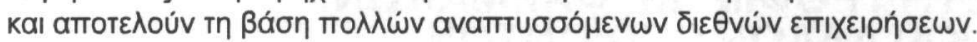

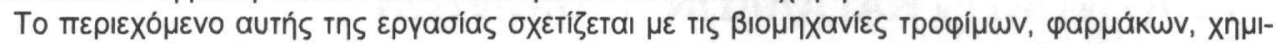

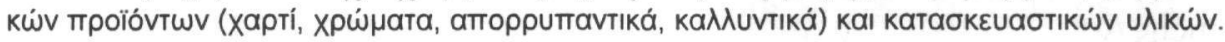

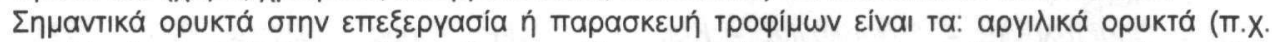

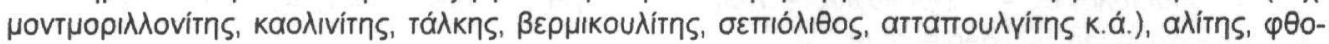

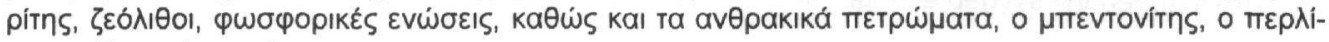

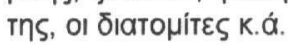

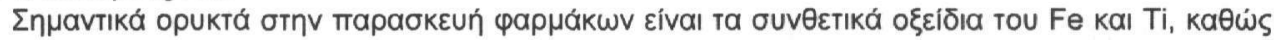

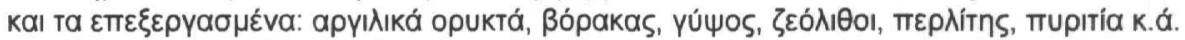

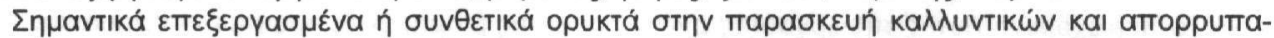

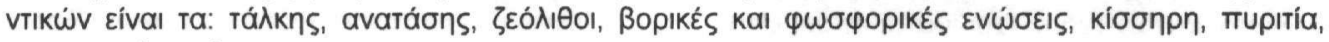

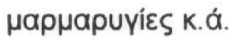

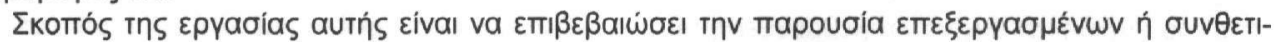

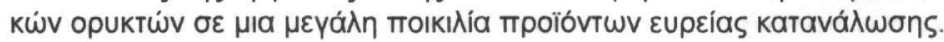

\section{$1.1 E \Delta \Omega \triangle I M A$ OPYKTA}

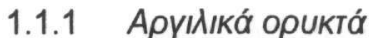

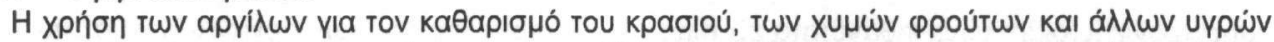

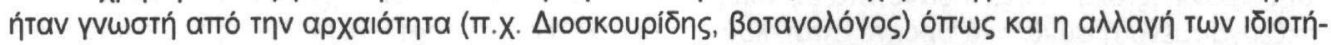

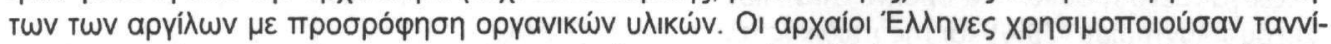

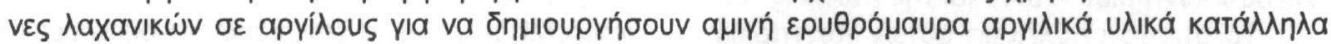

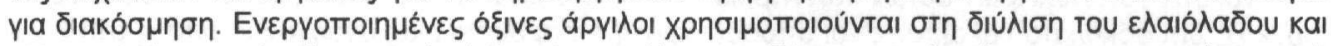

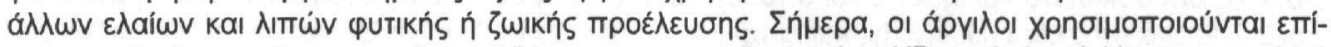

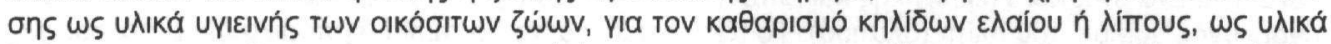

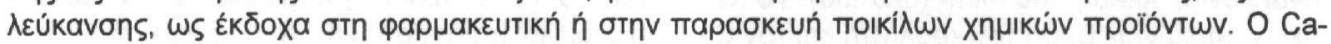

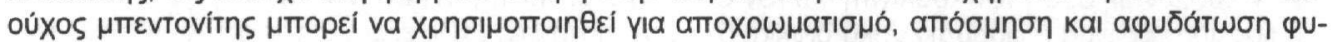

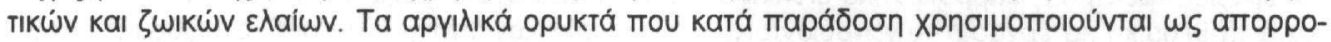

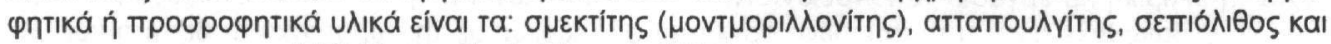

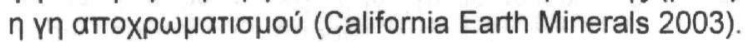

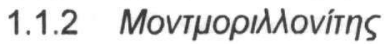

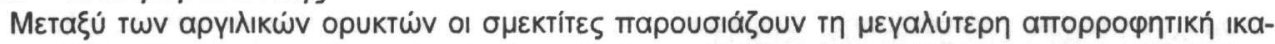

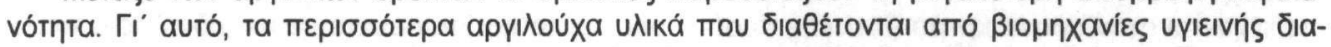

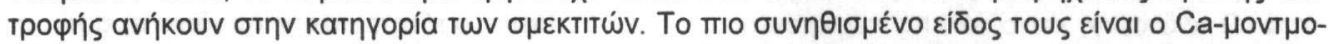

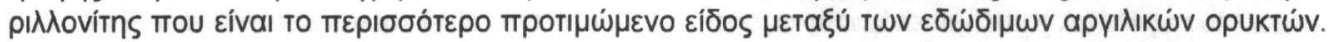

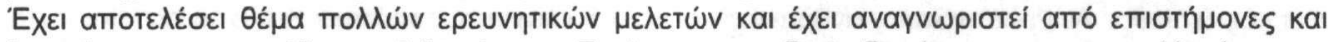

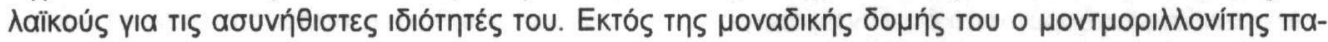

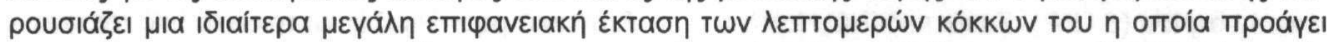

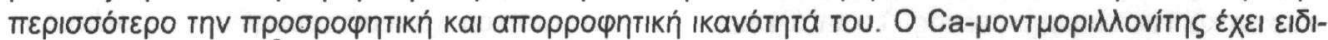

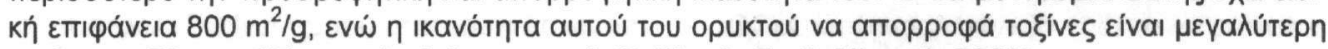

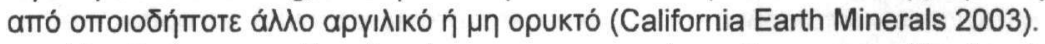

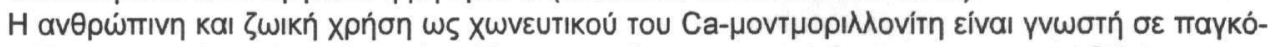

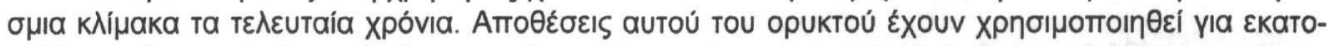

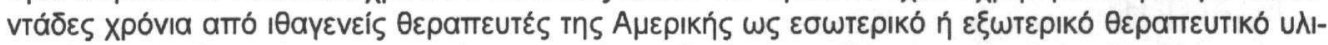

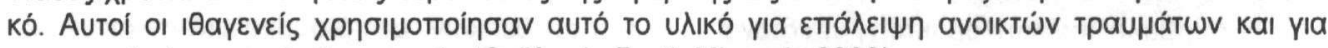

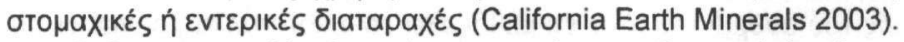

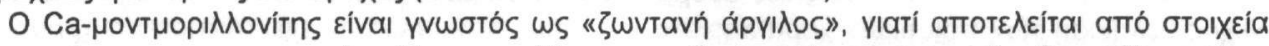

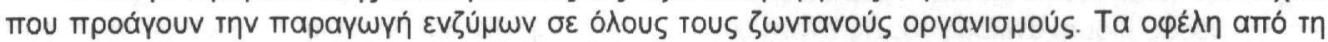

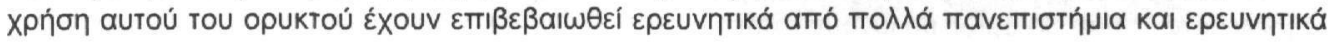




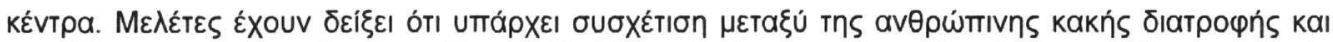

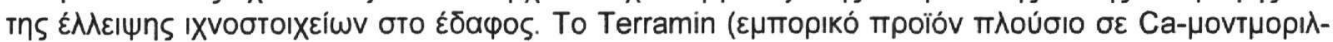

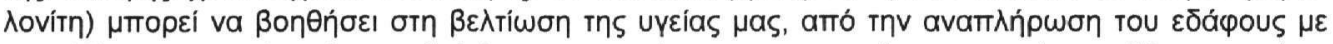

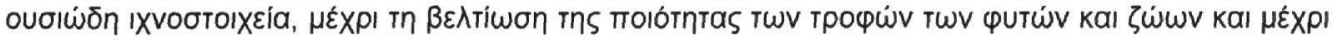

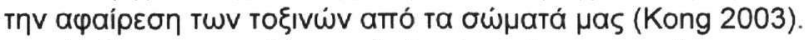

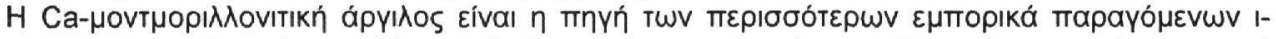

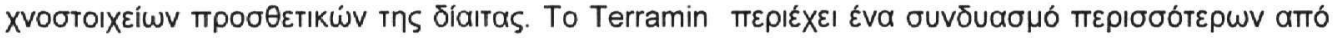

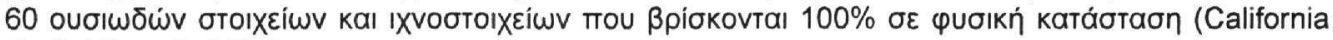
Earth Minerals 2003).

\subsubsection{Kooגivítns}

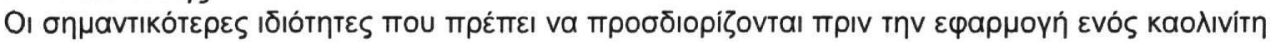

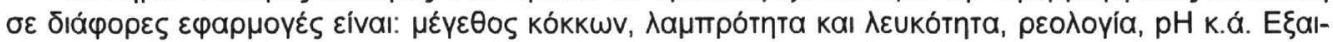

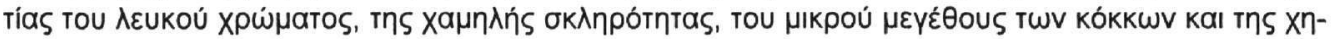

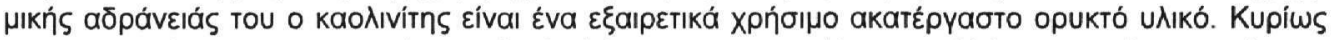

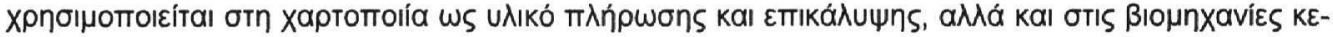

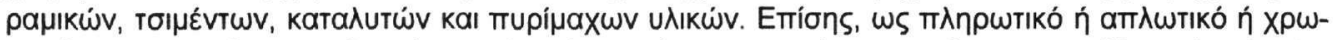

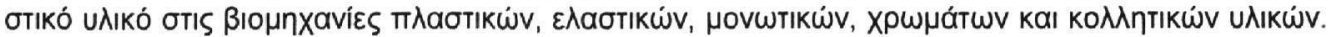

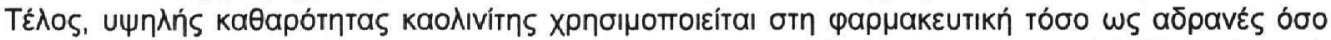

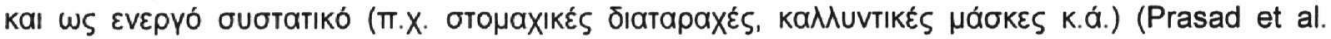
1991).

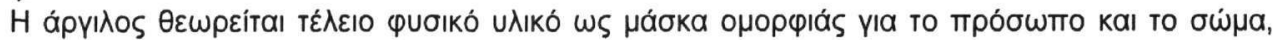

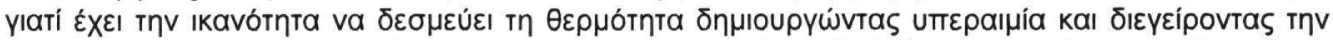

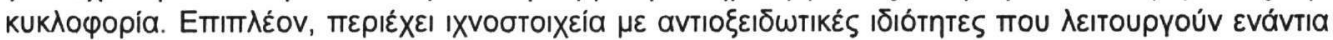

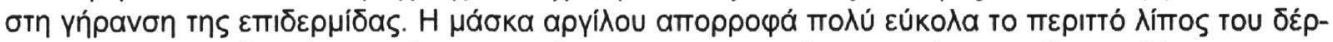

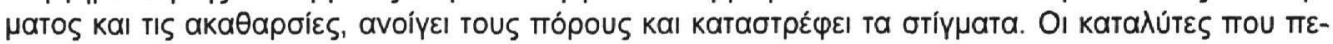

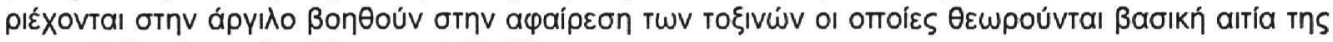
kuтTapírıסаs (www. freshline.co.uk 2003).

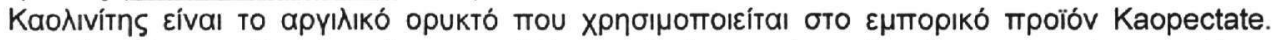

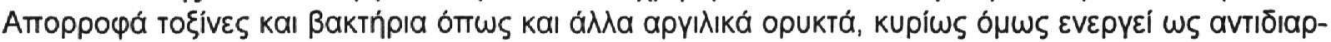

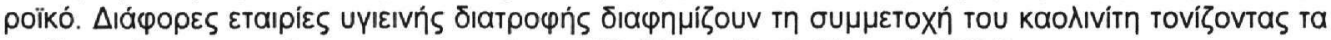

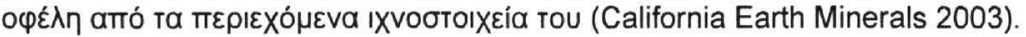

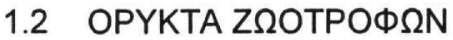

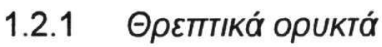

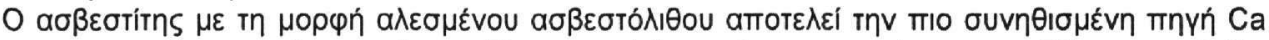

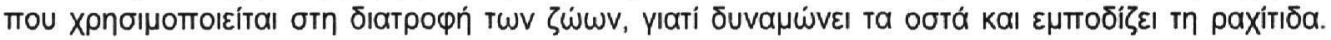

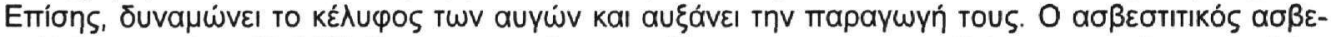

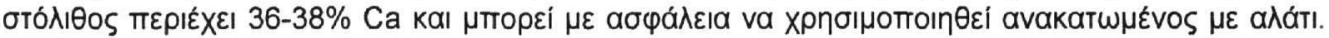

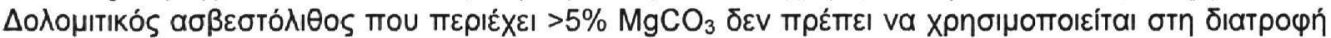

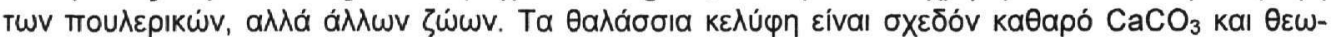

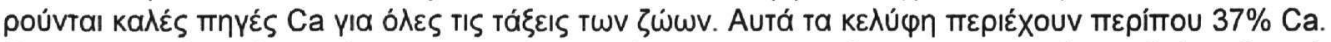

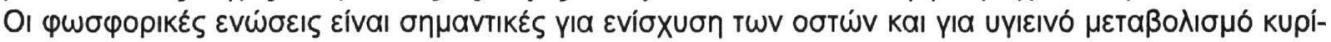

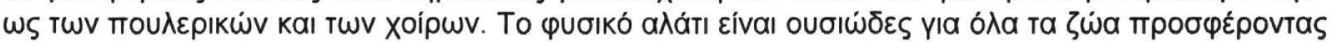

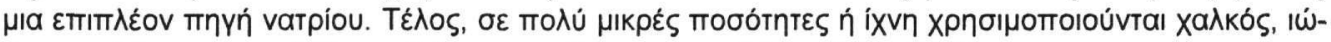

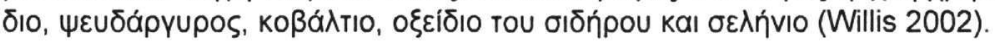

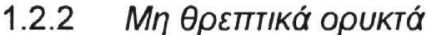

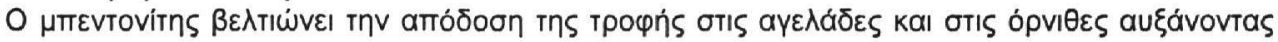

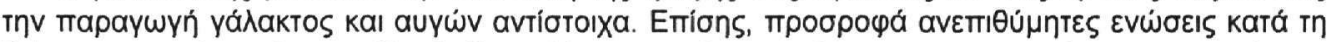

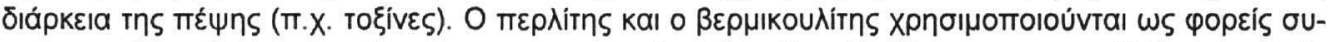

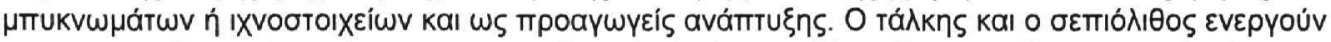

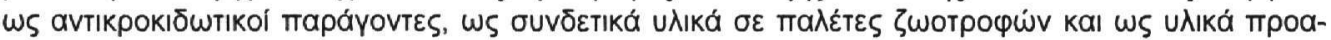

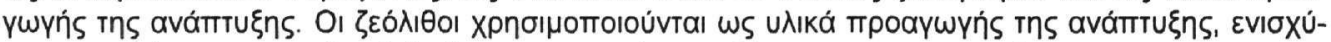

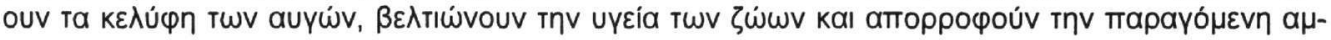

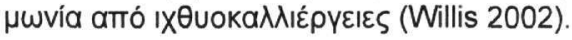




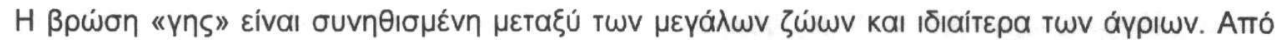

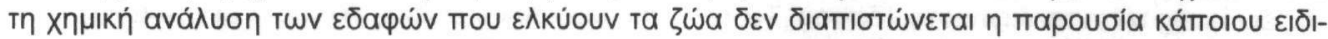

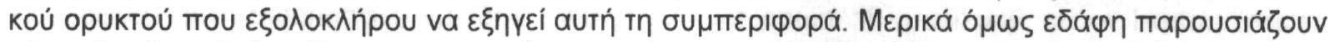

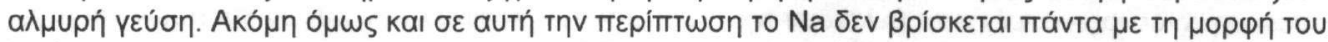

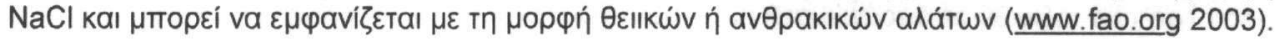

\section{3 ФОOРІTH $\Sigma$}

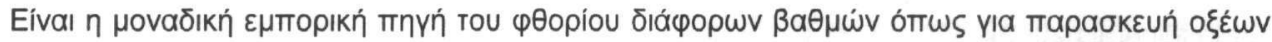

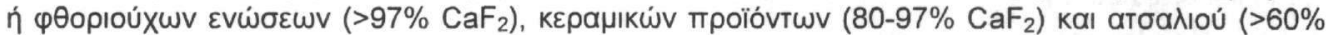
$\mathrm{CaF}_{2}$ ). Kovıото

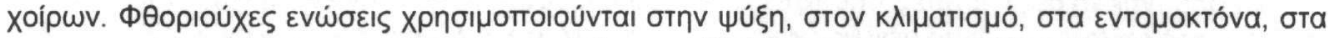

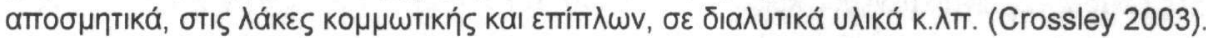

\section{$1.4 \quad \triangle \mathrm{IA \Sigma \Pi OPO}$}

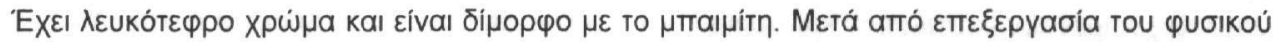

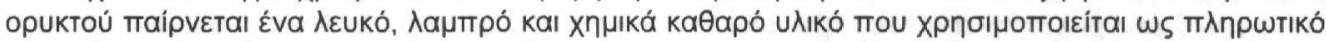

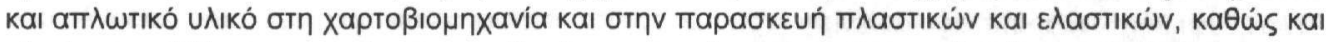

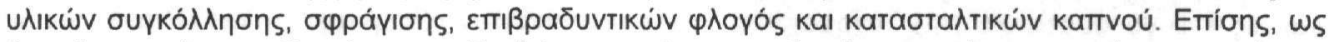

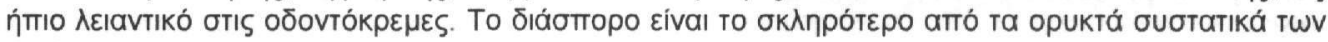

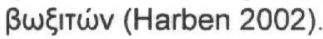

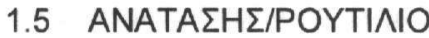

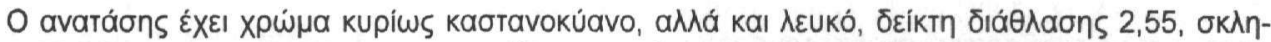

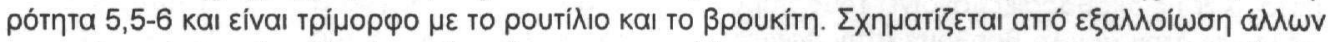

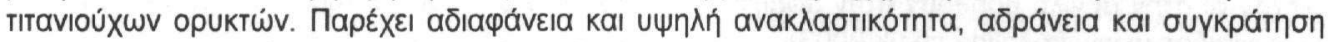

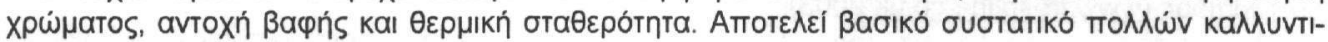
kúv (www. freshline.co.uk 2003).

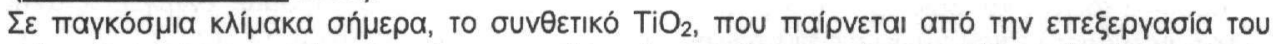

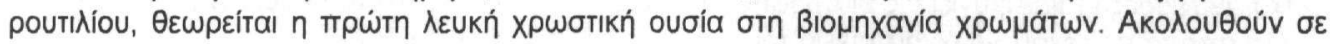

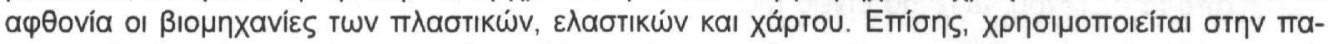

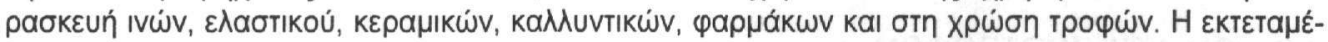

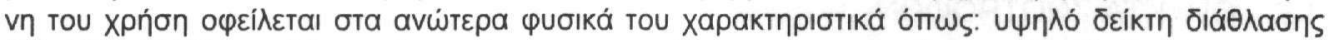

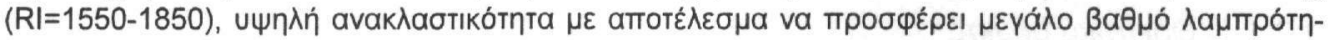

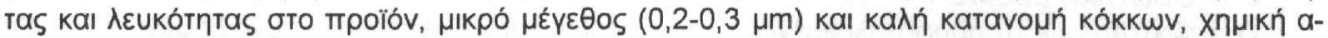

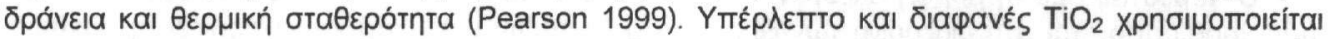

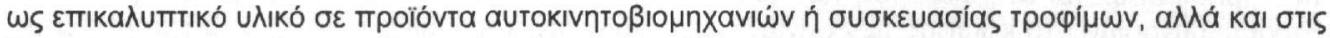

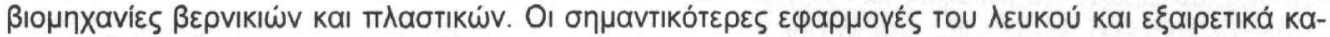

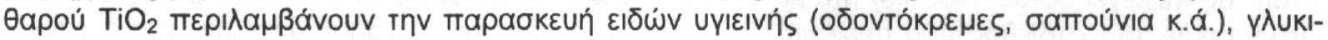

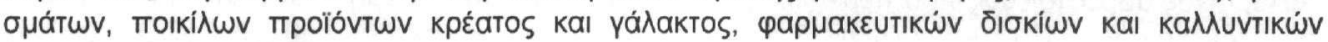
(www.kemira.com 2003).

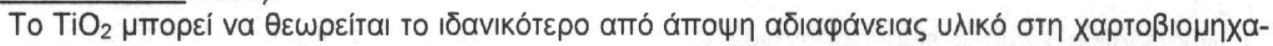

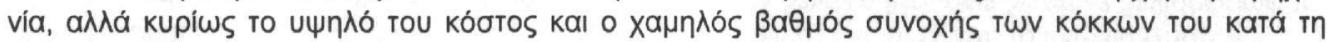

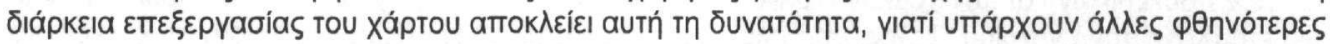

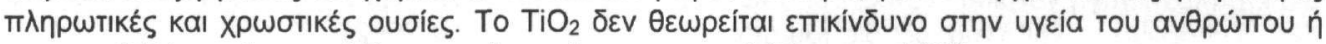

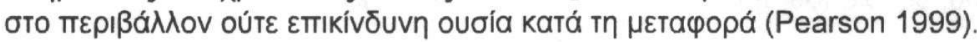

\section{$1.6 \mathrm{PO} \Delta \mathrm{OXP} \Omega \Sigma \mathrm{ITH} \Sigma$}

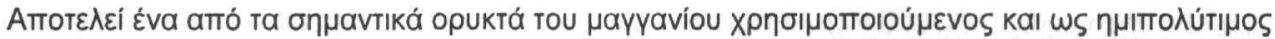

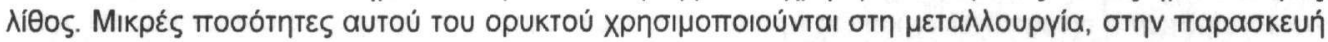

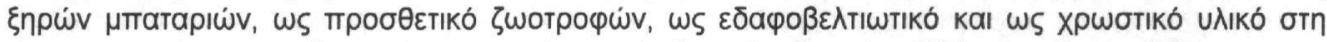

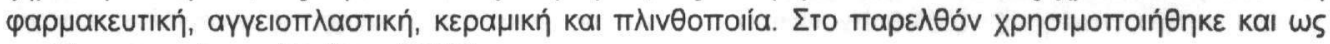

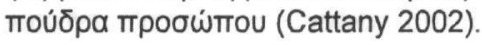




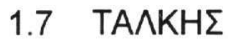

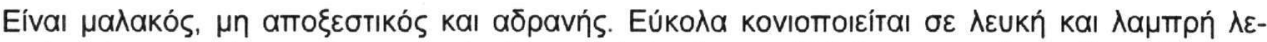

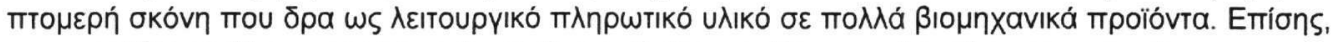

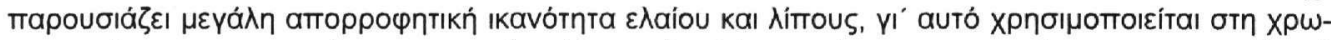

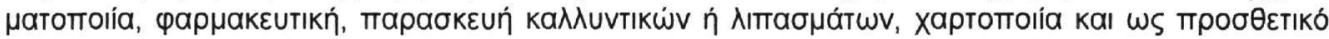

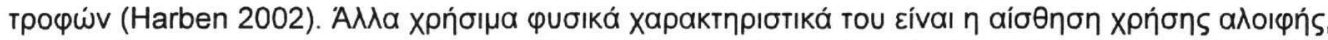

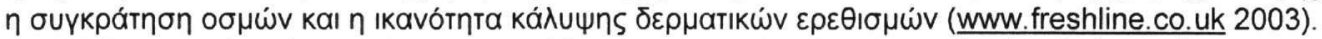

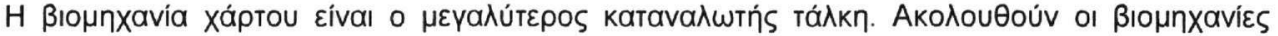

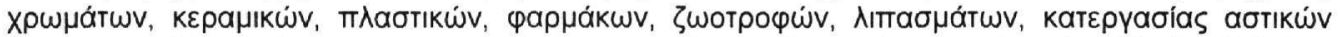

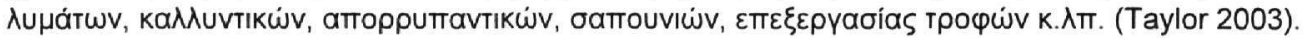

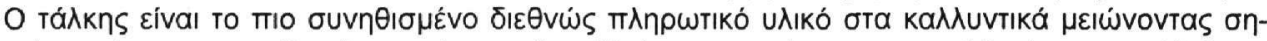

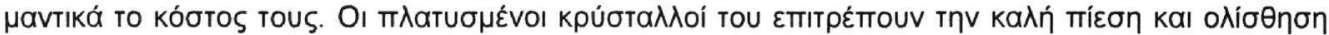

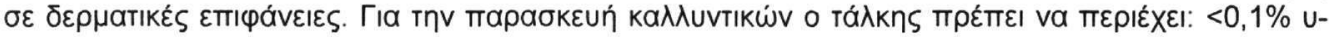

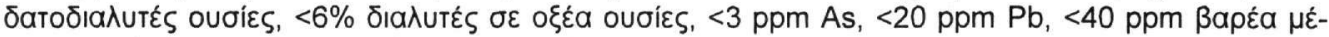

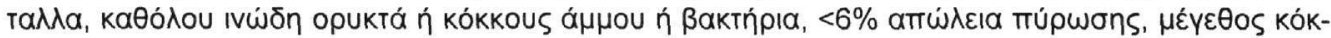
$\kappa \omega v<75 \mu \mathrm{m}$ ( $\mu \varepsilon \dot{\sigma o}$ ó ó $7 \mu \mathrm{m}$ ) kaı pH = 9,5 (Harben 2002).

\subsection{AIMATITH $\Sigma$}

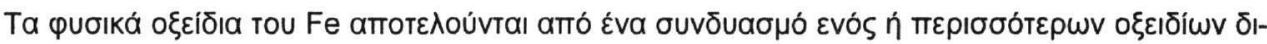

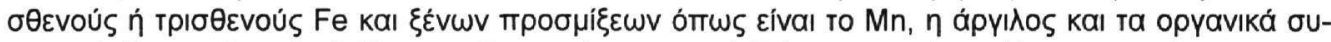

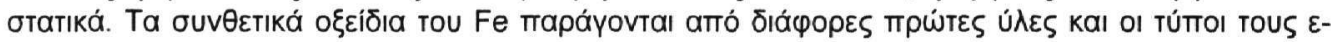

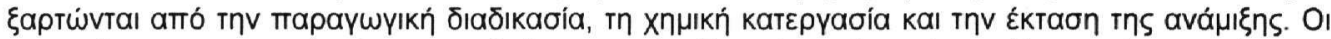

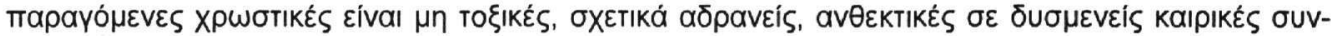

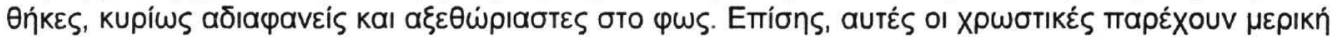

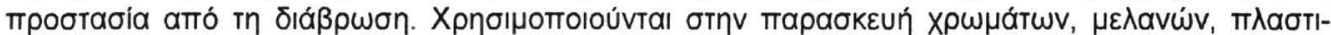

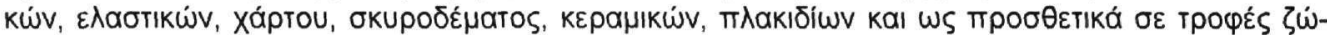

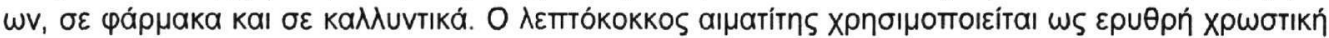

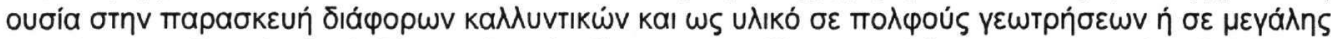

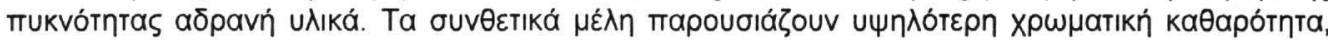

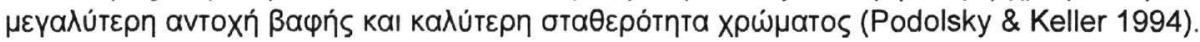

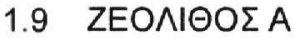

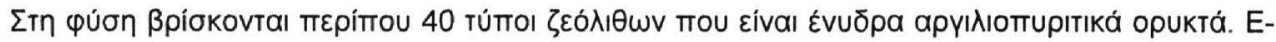

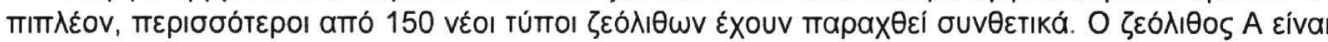

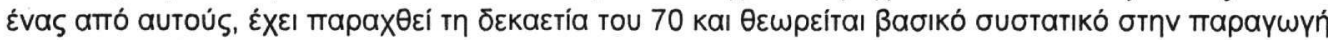

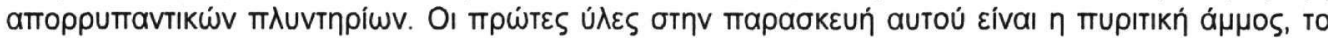
$\mathrm{NaCl}$ kaı o $\beta \omega \xi i$ ins (Fawer et al. 1998).

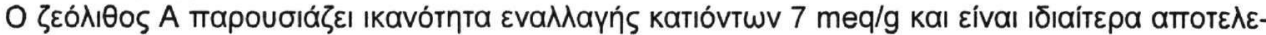

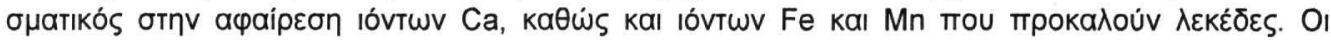

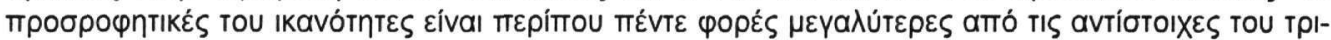

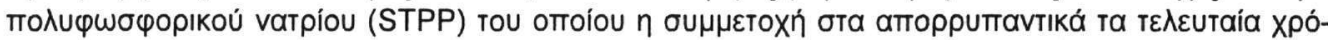

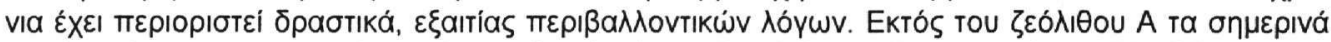

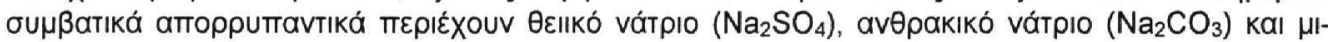

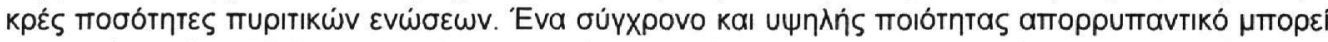

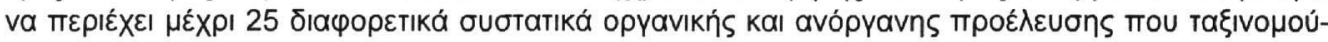

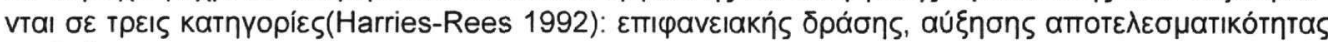
kaı $\lambda \varepsilon u ́ k a v \sigma n s$

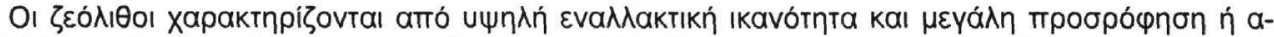

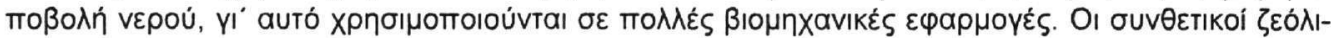

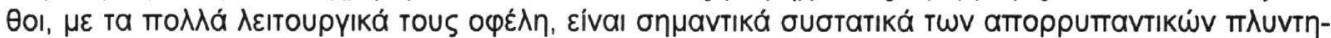

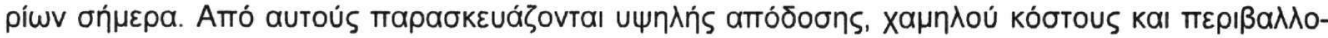

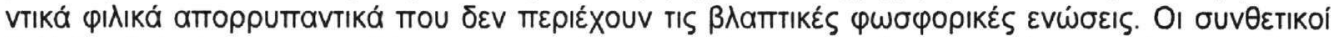

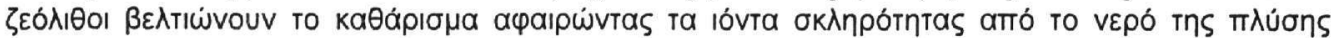
(www.pqcorp.com 2003). 


\subsection{ANOPAKIKO A $\Sigma B E \Sigma T I O$}

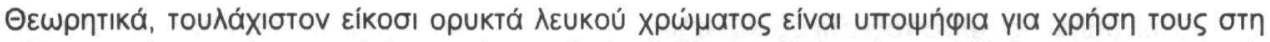

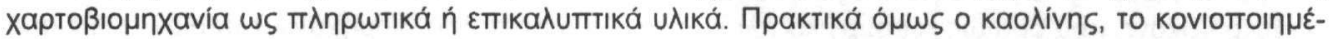

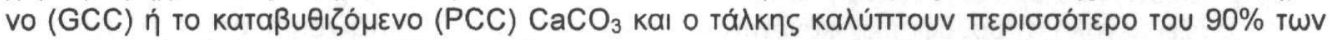

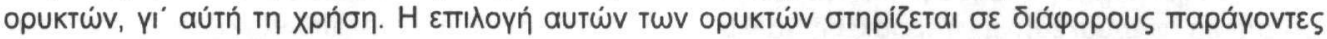

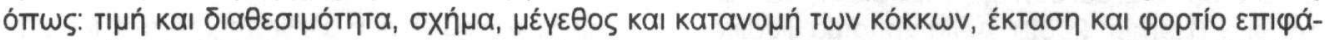

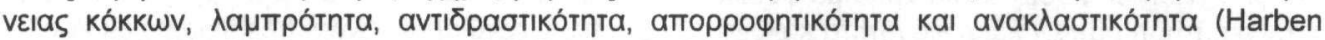

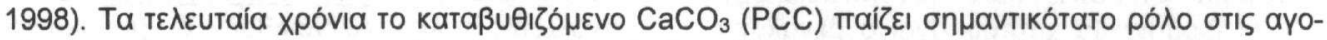

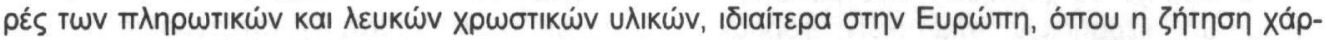

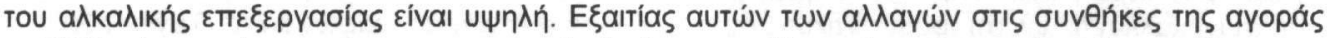

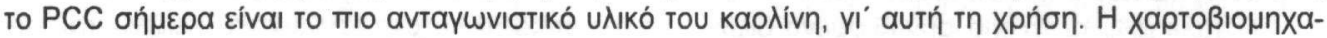

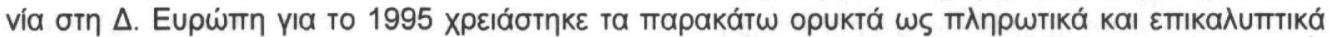

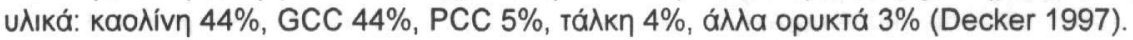

\subsection{MAPMAPYTIE $\Sigma$}

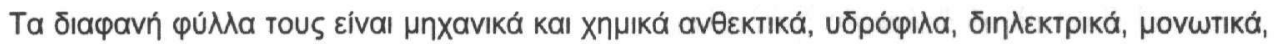

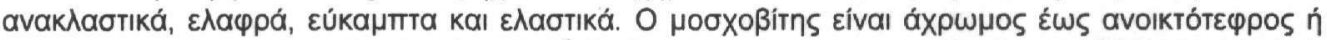

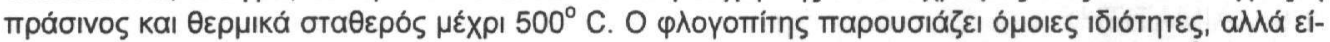

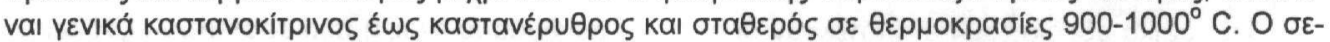

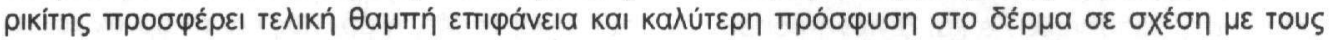

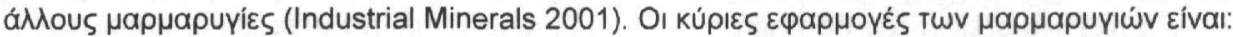

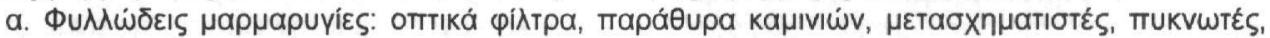

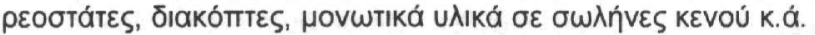

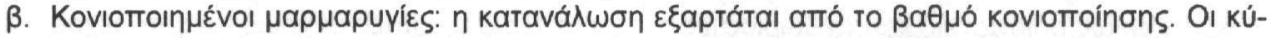

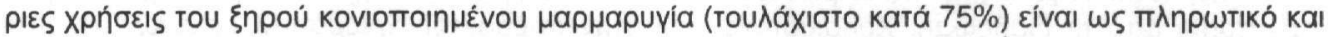
aтก

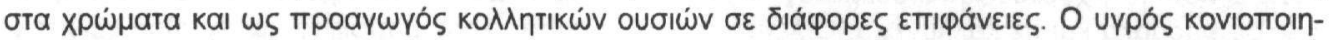

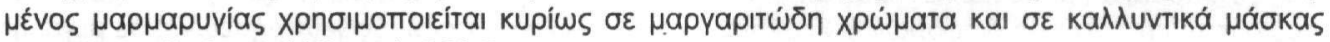
троби́тrou (Industrial Minerals 2001).

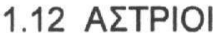

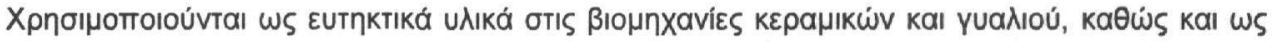

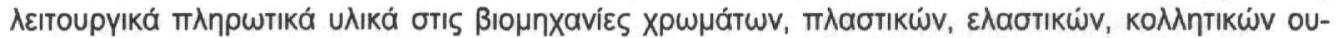

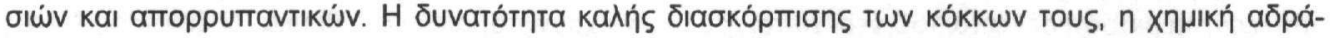

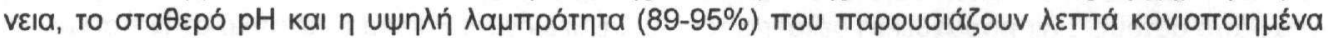

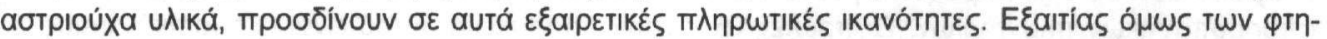

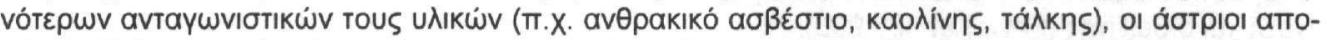

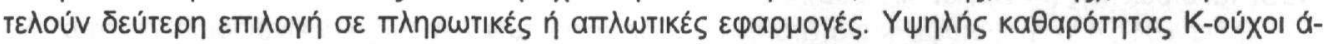

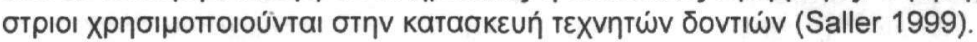

\section{YAIKA KAI ME $\Theta O \triangle O I$}

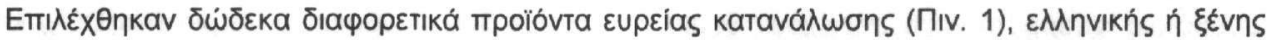

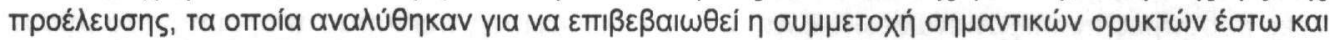

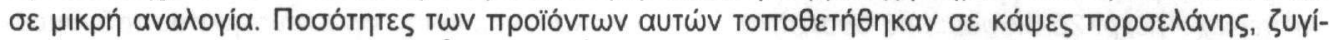

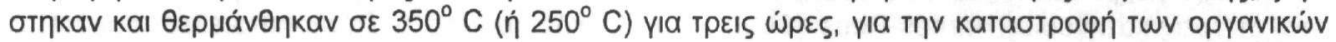

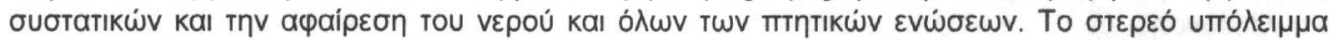

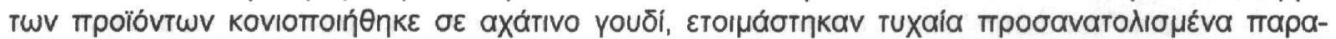

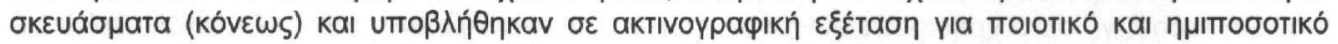

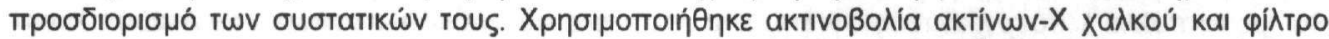

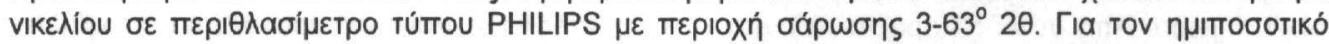

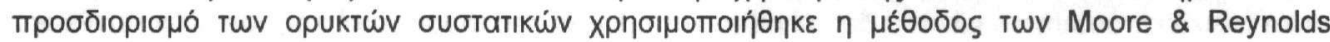
(1997). 


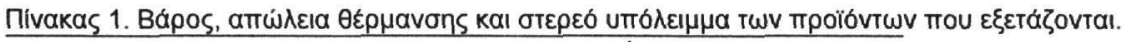

\begin{tabular}{|c|c|c|c|}
\hline חpoïóv & Bápos (g) & $\mathrm{LOH}^{1}(\mathrm{~g})$ & 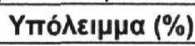 \\
\hline 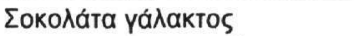 & 52,03 & 34,37 & 34 \\
\hline 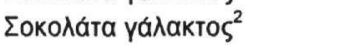 & 30,60 & 10,36 & 66 \\
\hline Mтাбкóra lomavías & 56,42 & 50,54 & 10 \\
\hline Мाтюко́та пт। $\mu \pi \varepsilon \rho^{2}$ & 28,06 & 22,47 & 20 \\
\hline Орvıөотрочń & 70,00 & 50,64 & 28 \\
\hline 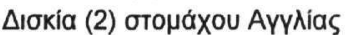 & 0,59 & 0,49 & 17 \\
\hline 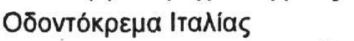 & 90,57 & 70,77 & 22 \\
\hline 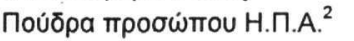 & 10,05 & 7,79 & 22 \\
\hline 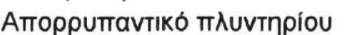 & 60,00 & 13,33 & 78 \\
\hline 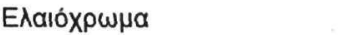 & 91,42 & 53,64 & 41 \\
\hline 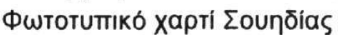 & 9,91 & 6,82 & 31 \\
\hline 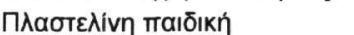 & 27,54 & 8,18 & 70 \\
\hline
\end{tabular}

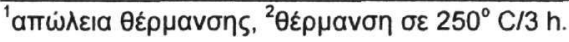

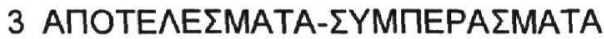

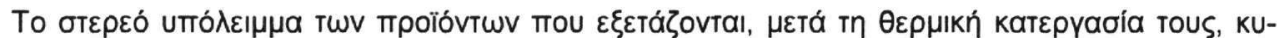

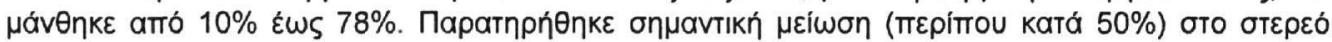

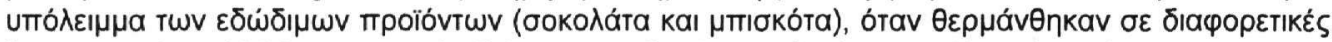

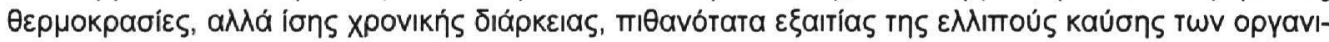

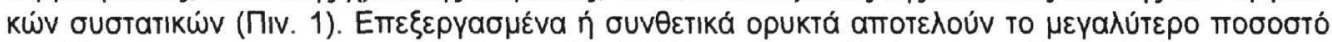

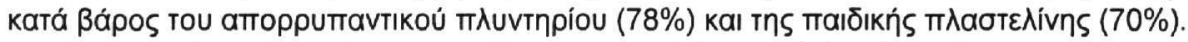

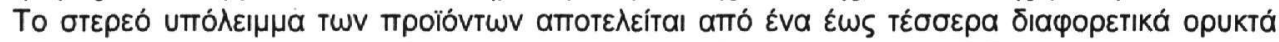

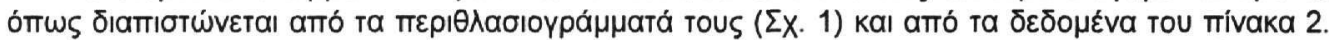

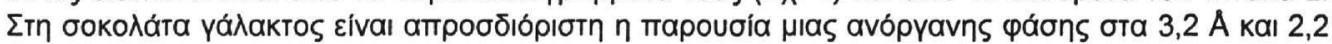

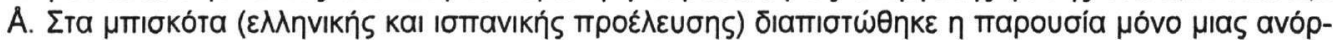

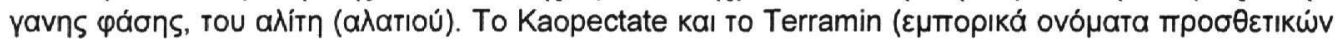

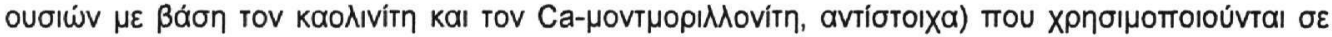

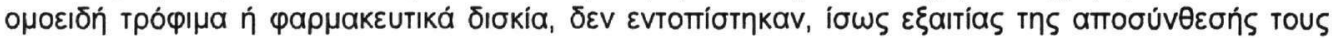

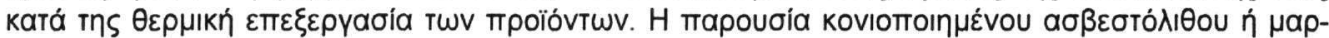

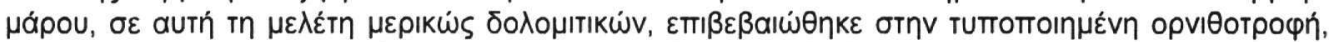

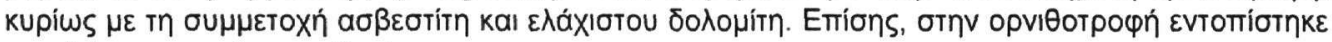

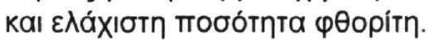

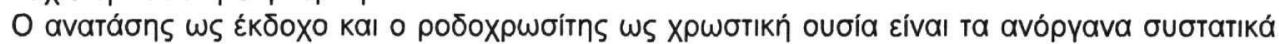

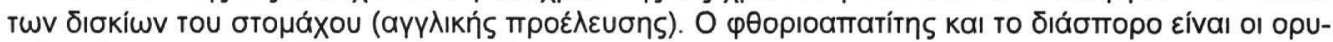

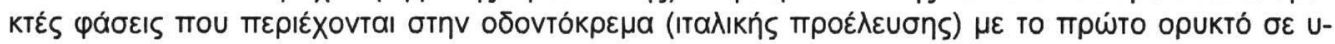

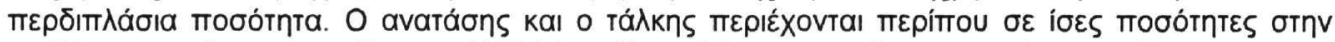

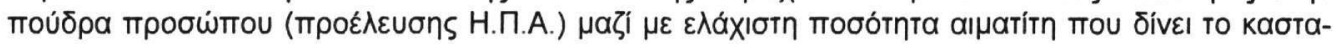

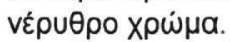

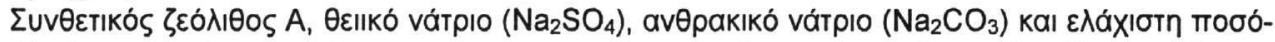

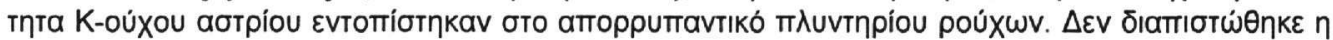

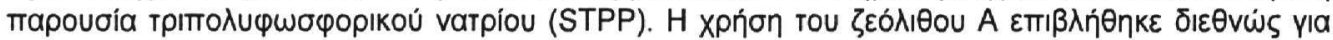

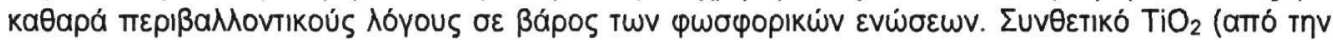

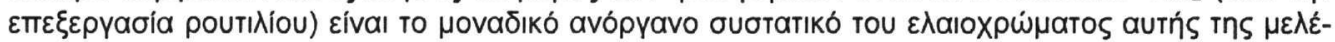
TnS.

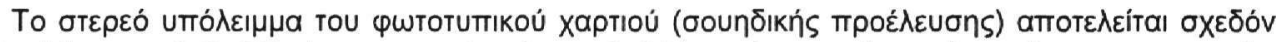

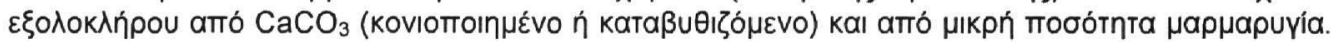

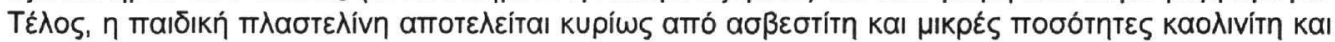

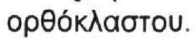



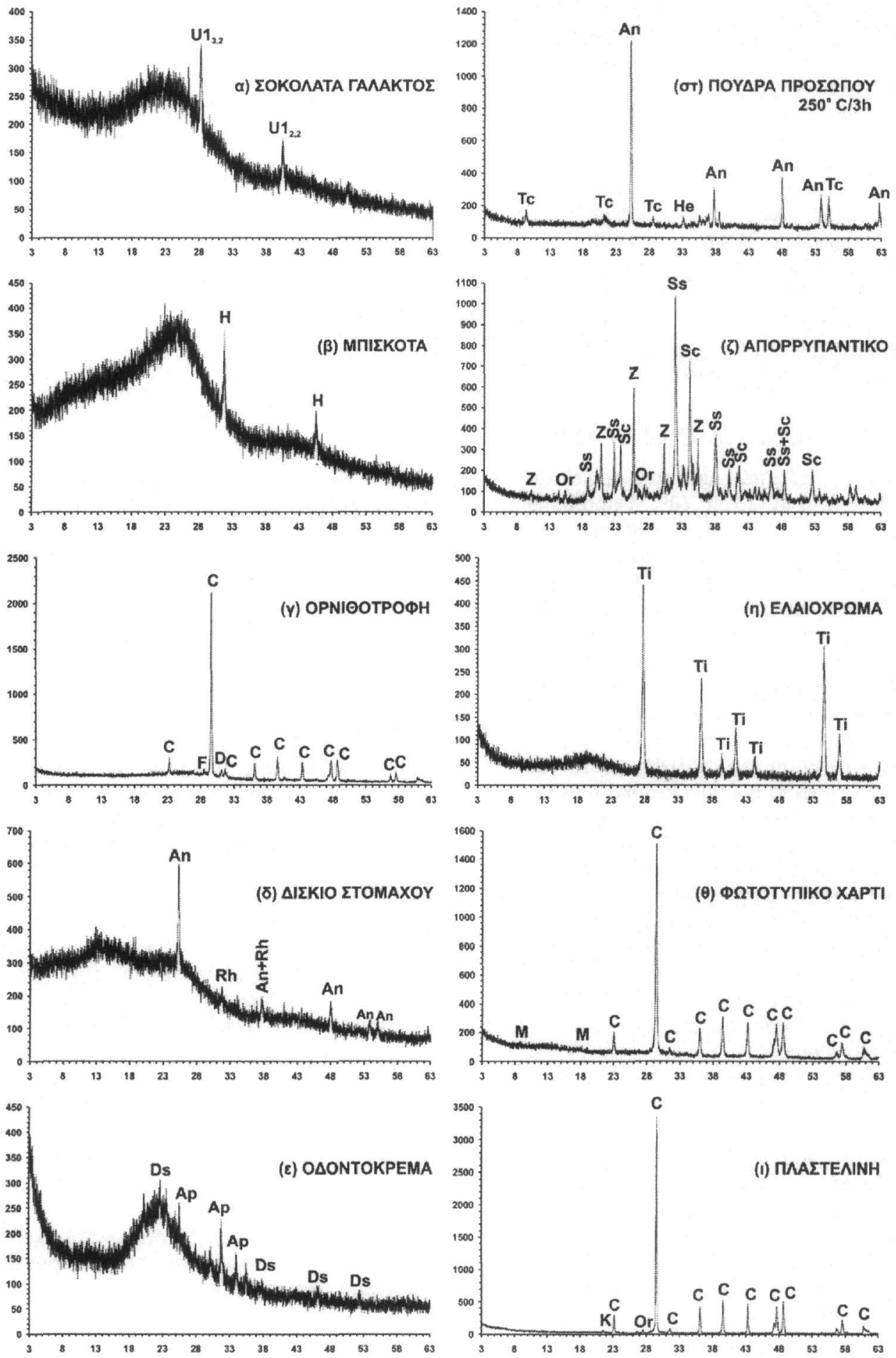

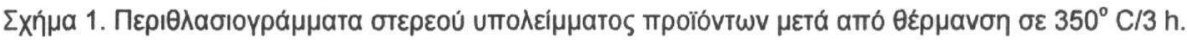

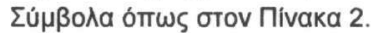




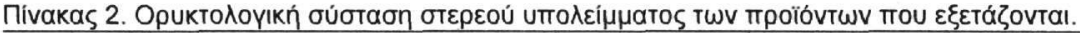

\begin{tabular}{|c|c|c|c|c|c|c|c|c|c|c|c|c|c|c|c|c|c|c|}
\hline Проїóv & $\mathbf{U}$ & $\mathrm{H}$ & Ap & Ds & An & Tc & $\mathrm{He}$ & $\mathbf{R h}$ & $\mathbf{Z}$ & Ss & Sc & $\mathrm{Ti}$ & C & D & $\mathbf{K}$ & Or & $\mathbf{F}$ & $\mathbf{M}$ \\
\hline 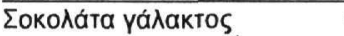 & +++ & & & & & & & & & & & & & & & & & \\
\hline 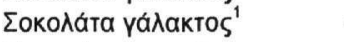 & +++ & & & & & & & & - & & & & & & & & & \\
\hline Мтıбко́та Іотаvías & & +++ & & & & & & & & & & & & & & & & \\
\hline Мтібко́та птІ $\mu т в \varepsilon \rho^{1}$ & & +++ & & & & & & & & & & & & & & & & \\
\hline OрvіӨотрофи́ & & & & & & & & & & & & & +++ & + & & & + & \\
\hline 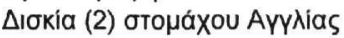 & & & & & +++ & & & + & & & & & & & & & & \\
\hline 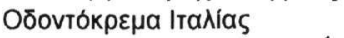 & & & ++ & ++ & & & & & & & & & & & & & & \\
\hline 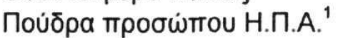 & & & & & ++ & ++ & + & & & & & & & & & & & \\
\hline Атторриттаvтіко́ плиvтпрі́ou & & & & & & & & & ++ & ++ & + & & & & & + & & \\
\hline Eגaı́x $\rho \omega \mu \alpha$ & & & & & & & & & & & & +++ & & & & & & \\
\hline 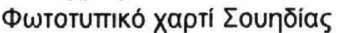 & & & & & & & & & & & & & +++ & & & & & + \\
\hline 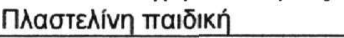 & & & & & & & & & & & & & +++ & & + & + & & \\
\hline
\end{tabular}

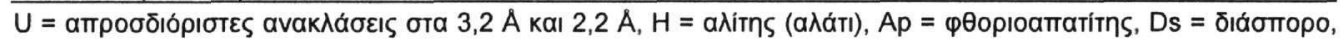

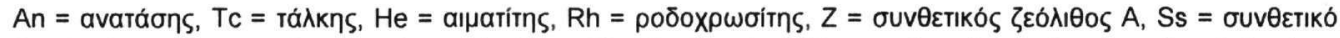

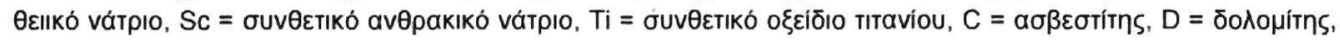

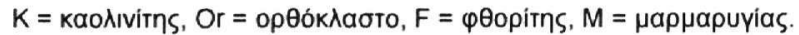

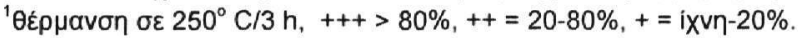

\section{ANAФOPE $\Sigma$}

California Earth Minerals Corp. 2003. California Earth Minerals. www.calearthminerals.com, 4p.

Cattany R.W. 2002. Over 100 Years Working for the Mining Industry in Colorado. Col. Mining Ass. Sp. Paper, $13 p$.

Crossley P. 2003. Fluorspar. Taking stock. Ind. Miner., 4, 46-53.

Decker M. 1997. Fillers and extenders: European market trends. Ind. Miner., 12, $29-35$.

Fawer M., Postlethwaite D. \& Kluppel H.-J. 1998. Life Cycle Inventory for the Production of Zeolite A for Detergents. Int. J. LCA, 3(2), 71-74.

Harben P. 1998. $\mathrm{CaCO}_{3}$ in paper: PCC versus the competition. Ind. Miner., 3, 39-49.

Harben P. 2002. The Industrial Minerals HandyBook. A guide to markets, specifications \& prices, $4^{\text {th }}$ ed. Ind. Miner. Inform., Surrey, UK, 412p.

Herries-Rees K. 1992. Minerals in detergents. Forever blowing bubbles. Ind. Miner., 11, 37-49.

Industrial Minerals 2001. Mica. Ind. Miner., 3, p19.

Kong R. 2003. Edible Clays. uww.the-vu.com, 3p.

Moore D.M. \& Reynolds R.C., Jr. 1997. X-ray Diffraction and the Identification and Analysis of Clay Minerals, $2^{\text {nd }}$ ed. New York, Oxford Univ. Press, 378p.

Pearson K. 1999. Pigment in the middle. A TiO 2 industry focus. Ind. Miner., 7, 56-69.

Podolsky G \& Keller D.P. 1994. Pigments, iron oxide. In: Carr D.D., (ed.), Industrial Minerals and Rocks, $6^{\text {th }}$ ed., Soc. Min. Metall. Expl., Littleton, Co, 765-781.

Prasad M.S., Reid K.J. \& Murray H.H. 1991. Kaolin: processing, properties and applications. App. Clay Sci., 6 , 87-119.

Saller M. 1999. Feldspar and nepheline syenite rewiewed. Ind. Miner., 10, 43-53.

Taylor L. 2003. Smooth operator. Talc gets specialised for growth. Ind. Miner., 5, 24-33.

Virta R., Lorenz W. \& Regueiro M. 1994. Industrial minerals and rocks: classification of end uses. Ind. Miner., 4, 65-67.

Willis M. 2002. From the horse's mouth. Ind. Miner., 11, 34-39.

www.fao.org. 2003. Animal Feed Resources Information System, 2p.

umw.freshline.co.uk 2003. Fresh Line, 48p. (in Greek).

www.kemira.com. 2003. Titanium Dioxide Pigments, 10p.

www.pqcorp.com. 2003. Detergents, $1 \mathrm{p}$. 


\section{THE USE OF PROCESSED OR SYNTHETIC MINERALS IN THE PREPARATION OF FOOD, PHARMACEUTICALS, COSMETICS AND OTHER PRODUCTS}

Tsirambides A.

Department of Mineralogy - Petrology - Economic Geology, School of Geology, A.U.Th., Thessaloniki 541 24, ananias@geo.auth.gr

The contribution of many minerals, processed or synthetic, in the industrial development and thus in the improvement of man's life has increased their use in new industrial sectors displacing other traditional products, mainly of organic origin which however contaminate the environment. The content of this paper is related to the industries of food, pharmaceuticals, chemicals (paper, paints, detergents, cosmetics) and building materials.

Twelve different products of wide consumption have been selected, of Greek or foreign origin, which were analysed in order to confirm the participation of common and special minerals even in small amounts. Quantities of these products were heated at $350^{\circ} \mathrm{C}$ (or $250^{\circ} \mathrm{C}$ ) for three hours in order to destruct the organic constituents and remove the water and all volatile compounds from them.

The solid residue of the studied products varied from $10 \%$ to $78 \%$. Great decrease (about $50 \%$ ) was noticed in the solid residue of the edible products (chocolate and biscuits) when they were heated at different temperatures of equal time duration, probably because of the incomplete burning of the organic constituents. Processed or synthetic mineral constitute the greater percentage by weight of the wash detergent $(78 \%)$ and the child's clay $(70 \%)$.

The solid residue of these products is composed of one to four different minerals. In the milk chocolate the presence of an inorganic phase at 3,2 $A$ and 2,2 $A$ is undetermined. In the biscuits (of Greek and Spanish origin) the presence of only one inorganic phase, halite, was confirmed. The Kaopectate and the Terramin, trade names of additives with base of kaolinite and Ca-montmorillonite respectively, which are used in similar products or pharmaceuticals, were not detected, probably because of their decomposition during the thermal processing of the products. The presence of ground limestone or marble in the chicken food was confirmed mainly with the participation of calcite and minor dolomite. In addition, in this food, minor amount of fluorite was detected, too. The anatase as carrier and the rhodochrosite as pigment are the inorganic constituents of stomach tablets (of English origin). Fluorapatite and diaspore are the mineral phases contained in the toothpaste (of Italian origin) with the first mineral in more than double amount. The anatase and the talc are contained about in equal amounts in the face make up (of USA origin) with minor hematite which gives the brown-red colour. Synthetic zeolite A, sodium sulphate, sodium carbonate and minor K-feldspar were detected in the wash detergent of cloths. The sodium tripolyphosphate (STPP) compound was not detected. The use of zeolite A was internationally imposed for environmental reasons against the phosphoric compounds. Synthetic $\mathrm{TiO}_{2}$ (from the rutile processing) is the unique inorganic constituent of the paint in this study. The solid residue of the phototypic paper (of Sweden origin) is composed almost completely of $\mathrm{CaCO}_{3}$ (GCC or PCC) and minor mica. Finally, the child's clay is mainly composed of calcite and minor amounts of kaolinite and orthoclase. 